\title{
The Biocultural Labels Initiative: Supporting Indigenous rights in data derived from genetic
}

\section{resources}

\author{
Jane Anderson‡, Maui Hudson§ \\ ‡ New York University / ENRICH, New York City, United States of America \\ $\S$ Te Kotahi Research Institute, University of Waikato / ENRICH, Hamilton, New Zealand
}

Corresponding author: Jane Anderson (ja77@nyu.edu)

Received: 01 Oct 2020 | Published: 09 Oct 2020

Citation: Anderson J, Hudson M (2020) The Biocultural Labels Initiative: Supporting Indigenous rights in data derived from genetic resources. Biodiversity Information Science and Standards 4: e59230.

https://doi.org/10.3897/biss.4.59230

\begin{abstract}
Internationally, questions of ethics and equity, especially in relation to responsible sharing of data across multiple national platforms, are creating a new range of issues for researchers in the science and innovation sectors. In many nation states, even for those that are not yet signatories to the Nagoya Protocol (Secretariat of the Convention on Biological Diversity 2011), there is a general acknowledgement that the future sharing of biological, ecological and environmental data in support of innovation and generative economic opportunities, must address the rights of Indigenous peoples. The Biocultural (BC) Labels and Biocultural (BC) Notices are two distinct tools delivered by the Local Contexts Platform for recognizing Indigenous rights in data derived from genetic resources. The key challenge being addressed by the Biocultural Labels initiative is how to practically encode Indigenous provenance information and cultural responsibilities into research data, including digital sequence information (DSI), on genetic resources. As a digital data ethics strategy, Biocultural Labels make visible the provenance and ethics of collections; outline community expectations and consents about appropriate use of the collections; connect data to people and environments, thereby maintaining relationships to data over time and enhancing the capacity for Indigenous control of Indigenous data.
\end{abstract}


Prioritizing provenance and transparency in data collected in collaboration and partnership with Indigenous peoples or on Indigenous lands and waters, the six Biocultural Labels provide a mechanism for Indigenous communities to directly integrate associated responsibilities, obligations and relationships as accompanying metadata. For example, the Provenance BC Label enables origin information, including Indigenous names and places to become metadata for data generated from Indigenous lands and/or waters; the Open to Collaboration BC Label indicates that an Indigenous community is open to future research collaborations and outreach activities that might arise from research relating to specific biodiversity data; the Consent Verified BC Label indicates that there are consent conditions in place from the Indigenous community for the data that have been generated from research; the Multiple Community BC Label recognizes that more than one community has responsibilities and interests in data about a particular species; the Research Use BC Label helps clarify that a community agrees that certain data may be used openly and without further negotiation for future research opportunities; and, the Open to Commercialization BC Label indicates that a community is open to the possibility of profit oriented opportunities arising from specific research or from specific species. The Biocultural Labels allow for cultural protocols to be digitally expressed and connected with data, travelling with it over time, by enhancing engagement with, connection to and recognition of Indigenous interests in biodiversity research. As a complementary, yet distinct mechanism, the Biocultural Notices are a tool for researchers and institutions to use and apply to data when underlying Indigenous rights or interests in collected data can and need to be identified. This mechanism helps increase researcher integrity when working with Indigenous communities, and generates transparency across the Indigenous data lifecycle. The BC Labels and Notices have been developed as a direct Ethical, Legal and Social Implications (ELSI) intervention for the equitable and ethical future use of Indigenous data. This initiative offers the possibility for substantive change in how biological data from Indigenous contexts can maintain cultural relationships and responsibilities, connecting Indigenous people and places over time with data and in the metadata and with future researchers for the cultural, ecological and commercial benefit of Indigenous peoples.

With the development of these digital tools, questions about standards and appropriate metadata fields for the Labels and Notices within the Darwin Core (DwC) standard, which incorporates previously defined Dublin Core (dcterms) terms, has arisen. This is partly because both the BC Labels and the BC Notices could potentially be used at a record level in multiple ways, for instance as dcterms:rightsHolder, dcterms:accessRights and potentially dcterms:license. It is also possible, that given the absence of Indigenous interests in the larger formation of the DwC standard, that something new might need to be created that can adequately represent these purpose and use interests, and the integrity in research practice that they promote. This panel brings together the founders of the Biocultural Labels initiative with users of the Labels within researcher and institutional contexts to discuss development, uptake, scaling, standards and appropriate metadata fields for the Labels and Notices within Genomic Standards Consortium standards, and TDWG Community Interest Groups (IG) including Attribution, Darwin Core, Genomic 
Biodiversity, Collections Descriptions and Species Information alongside TDWG Task Groups (TG) including Vocabularies and People in Biodiversity Data.

\section{Keywords}

Biocultural Labels and Notices, metadata, standards, ELSI, Dublin Core Metadata Initiative, DCMI, Darwin Core

\section{Presenting author}

Jane Anderson, Maui Hudson.

\section{Presented at}

TDWG 2020

\section{Grant title}

Te Tuākiri o te Tāonga: Recognizing Indigenous Interests in Genomic Research

\section{Conflicts of interest}

None

\section{References}

- Secretariat of the Convention on Biological Diversity (2011) Nagoya Protocol on Access to Genetic Resources and the Fair and Equitable Sharing of Benefits Arising from their Utilization to the Convention on Biological Diversity. URL: https://www.cbd.int/abs/doc/ protocol/nagoya-protocol-en.pdf 